\title{
Intermanual transfer effects in sequential tactuomotor learning: evidence for effector independent coding
}

Citation for published version (APA):

van Mier, H. I., \& Petersen, S. E. (2006). Intermanual transfer effects in sequential tactuomotor learning: evidence for effector independent coding. Neuropsychologia, 44, 939-949.

https://doi.org/10.1016/j.neuropsychologia.2005.08.010

Document status and date:

Published: 01/01/2006

DOI:

10.1016/j.neuropsychologia.2005.08.010

Document Version:

Publisher's PDF, also known as Version of record

Document license:

Taverne

Please check the document version of this publication:

- A submitted manuscript is the version of the article upon submission and before peer-review. There can be important differences between the submitted version and the official published version of record.

People interested in the research are advised to contact the author for the final version of the publication, or visit the DOI to the publisher's website.

- The final author version and the galley proof are versions of the publication after peer review.

- The final published version features the final layout of the paper including the volume, issue and page numbers.

Link to publication

\footnotetext{
General rights rights.

- You may freely distribute the URL identifying the publication in the public portal. please follow below link for the End User Agreement:

www.umlib.nl/taverne-license

Take down policy

If you believe that this document breaches copyright please contact us at:

repository@maastrichtuniversity.nl

providing details and we will investigate your claim.
}

Copyright and moral rights for the publications made accessible in the public portal are retained by the authors and/or other copyright owners and it is a condition of accessing publications that users recognise and abide by the legal requirements associated with these

- Users may download and print one copy of any publication from the public portal for the purpose of private study or research.

- You may not further distribute the material or use it for any profit-making activity or commercial gain

If the publication is distributed under the terms of Article $25 \mathrm{fa}$ of the Dutch Copyright Act, indicated by the "Taverne" license above, 


\title{
Intermanual transfer effects in sequential tactuomotor learning: Evidence for effector independent coding
}

\author{
Hanneke I. van Mier ${ }^{\mathrm{a}, *}$, Steven E. Petersen ${ }^{\mathrm{b}, \mathrm{c}, \mathrm{d}, \mathrm{e}}$

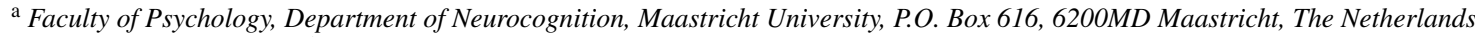 \\ ${ }^{\mathrm{b}}$ Department of Radiology, Washington University, St. Louis, MO 63130, USA \\ ${ }^{c}$ Department of Neurology and Neurological Surgery, Washington University, St. Louis, MO 63130, USA \\ ${ }^{\mathrm{d}}$ Department of Anatomy and Neurobiology, Washington University, School of Medicine, St. Louis, MO 6310, USA \\ e Department of Psychology, Washington University, St. Louis, MO 63130, USA
}

Received 29 March 2005; received in revised form 18 August 2005; accepted 23 August 2005

Available online 29 September 2005

\begin{abstract}
Results from our earlier brain imaging studies regarding motor learning have shown different areas activated during naive and practiced performance. When right handed participants moved a pen either with the dominant or non-dominant hand continuously through a cut-out maze as quickly and accurately as possible, practice resulted in decreased brain activity in right premotor and parietal areas as well as left cerebellum, while increased activity was found in the supplementary motor area (SMA). These lateralized practiced-related changes in brain activation suggest effector-independent abstract coding of information.

To test this hypothesis more extensively, intermanual transfer of learning was examined in 24 male and female participants (12 right- and 12 left-handed) using the same maze-learning task. It was hypothesized that if an abstract representation of the movement is learned and stored, intermanual transfer effects should be more pronounced when participants transferred to a same maze as opposed to a mirror image of the maze. Errors and velocity were measured during the following conditions: initial naive performance (Naive); after practice on the maze (Prac); during intermanual transfer to the same maze (Transfer Identical); and to the mirror maze (Transfer Mirror). Transfer direction was tested from the dominant to non-dominant hand and vice versa.

No significant differences were found between right- and left-handed participants, males and females, and transfer directions. However, intermanual transfer of learning was significantly greater to the identical maze as opposed to the mirror maze. These results showed that learning was indeed taking place at an abstract effector independent level.
\end{abstract}

(C) 2005 Elsevier Ltd. All rights reserved.

Keywords: Motor skill learning; Transfer symmetry; Sequence; Handedness; Gender

\section{Introduction}

Practice makes perfect. It is common knowledge that for most tasks one has to practice in order to become skillful at that task. When performing a motor task for the first time, performance is often slow, inaccurate and uncoordinated. It is usually only after practice that fast, accurate and coordinated motor performance is observed. Recent neuroimaging research has shown that these behavioral changes are accompanied by changes in brain activations. Several imaging studies

\footnotetext{
* Corresponding author. Tel.: +31 43 3884010; fax: +31 433884125 .

E-mail address: h.vanmier@psychology.unimaas.nl (H.I. van Mier).
}

observed a shift in activated areas between unskilled and more skilled performance in motor tasks (Petersen, van Mier, Fiez, \& Raichle, 1998; Sakai et al., 1998; Van Mier, Tempel, Perlmutter, Raichle, \& Petersen, 1998; Van Mier, Perlmutter, \& Petersen, 2004; Doyon, Penhune, \& Ungerleider, 2003).

Remarkable is that after practice not only the trained limb performs more or less 'perfectly', in most cases also the untrained limb shows improvement, illustrating one of the mechanisms that might play a role in highly flexible motor behavior often seen in humans. In situations where we are not able to use our preferred hand, we can usually use our other hand or even another limb (think about (un)locking your door with the nondominant hand or closing a door with the shoulder or foot). This 
phenomenon of transfer might be utilized when designing training or rehabilitation programs.

The organization of motor control is often assumed to be hierarchical with an abstract effector-independent level constraining lower effector-dependent levels (Rosenbaum, Kenny, \& Derr, 1983; Van Mier \& Hulstijn, 1993; Tresilian, 1999). A central abstract representation is suggested consisting of a common description of the movement in such a way that the movement can be executed with different effectors (e.g. right versus left hand) or different muscles of the same effector (e.g. right hand versus right arm). With reference to effector independence, the handwriting study by Raibert (1977) is often cited as empirical support of an abstract representation. In his study, Raibert wrote the same sentence with different effectors. He found that regardless of the effector used (right hand, right arm, left hand, mouth or foot) the overall pattern of handwriting was the same. The shape of the letters was invariant in all writing conditions. Wright (1990), however, showed that although the overall shape of the letters was very similar, marked differences between effectors were apparent, indicating a certain level of effector dependency as well.

Using positron emission tomography (PET) to study changes in brain activations during motor sequence learning, Van Mier et al. (1998) scanned 32 right-handed participants while tracing a cut-out maze design with eyes closed during naive as well as practiced performance. In this study a switch in brain activations from unskilled to skilled performance was observed, with right premotor and parietal regions as well as left cerebellum significantly more activated during naive, unskilled tracing while activation in the supplementary motor area (SMA) was significantly higher after practice when skilled tracing was achieved. When participants were presented after practice with a novel maze (in this experiment a $90^{\circ}$ counterclockwise rotation of the practiced maze) performance returned to levels comparable during naive tracing. This slower and less accurate performance was accompanied by increased activation in right premotor and parietal regions and left cerebellum, the same areas that showed increased activation in the naive condition. Skilled performance and SMA activation seemed specific to the practiced maze sequence.

Another important finding reported in that study was the observation that the activations related to the level of skill or practice were seen in the same hemisphere and areas regardless of the hand that was used to perform the task. In the experiment, half of the participants performed the task with their dominant right hand, the other half with their non-dominant left hand. Both groups showed the same practice related changes (e.g., decreased blood flow in right premotor cortex, right parietal regions, and left cerebellum and increased blood flow in SMA after practice). These lateralized practice related changes suggest that the areas involved in this maze learning task process information at an effector independent abstract level, rather than at a motor execution or effector level (e.g. muscle agonistantagonist relationships, joints, etc.). In a follow up study involving the same maze-learning task, 16 right-handed participants were scanned during the course of practice (Van Mier et al., 2004). Results showed that decreases and increases in above- mentioned brain areas showed different learning curves and correlated significantly with changes in particular behavioral variables.

In order to perform the above-mentioned maze task skillfully and smoothly, the correct sequence of the maze segments has to be learned (Van Mier, Hulstijn, \& Petersen, 1993). Van Mier et al. (1993) have shown that during early performance these segments were performed as single units, while with repeated practice of the sequence these segments were more and more concatenated and performed as one unit. With the SMA becoming and being more activated during and after practice in our imaging studies (Van Mier et al., 1998, 2004), it seems that when learning is acquired, representation of the movement sequence appears in this area. This idea is consistent with results from other imaging studies (Rao et al., 1993; Grafton, Hazeltine, \& Ivry, 1998) as well as results from single unit recording studies (Tanji \& Shima, 1994), showing SMA activation related to sequential aspects of a task. Furthermore, transcranial magnetic stimulation (TMS) over the SMA interfered with normal improvement during practice of key press sequences (Verwey, Lammens, \& Honk, 2002). Grafton et al. (1998) found increased SMA activation during learning of a key press task performed with the right dominant hand, as well as with the left nondominant hand (Grafton, Hazeltine, \& Ivry, 2002). Furthermore, in the former study (Grafton et al., 1998) intralimb transfer of the same task to right arm performance activated the SMA to almost the same extent. For an overview of imaging studies showing SMA involvement in motor learning see Van Mier (2000).

Effector independent representations are also reported in dorsal premotor cortex (Tanji, Okano, \& Sato, 1988; Cisek, Crammond, \& Kalaska, 2003). Recently, evidence from patients with damage to unilateral sensorimotor cortex suggests that sensorimotor areas may also contribute to abstract representations (Hazeltine, 2001).

The lateralized practice related changes found in our study (Van Mier et al., 1998) suggest that what is stored is most likely the abstract movement sequence of the maze path. The purpose of this study was to test this hypothesis more extensively by having right and left-handed participants learn mazes with their dominant as well as non-dominant hand and examine intermanual transfer of learning to either the same maze or the mirror image of the maze. Keeping the movement sequence the same by having participants trace the same maze path with the opposite hand was expected to show more transfer effects than having participants trace the mirror image of the maze, in which the movement sequence is mirrored around the vertical axis. In the former, the same abstract movement sequence can be used and accessed with either the right or left hand. However, in the case of intermanual transfer to the mirror maze a new sequence has to be learned, and therefore no or less transfer to this maze is anticipated.

When a gain or loss in performance on one task as a result of practice on another task is observed, this is defined as transfer or interference of learning (Schmidt \& Lee, 1999). It involves the effect of experience in a prior task on the performance or learning of novel tasks. In a study by Fischman and Lim (1991), interference from practice on one- and two-target-striking 
responses to three-target responses was exhibited during transfer performance with the right as well as left hand. Remarkably, although only the right hand had performed the one- and twotarget responses, bilateral transfer of interference was observed, suggesting that the same abstract representation was used to produce movements by either hand.

Inter- or intralimb transfer of learning refers to situations in which practice of a certain motor task with a particular limb results in increased proficiency in performance on the same task with a different or contralateral limb without any previous involvement of the latter in task acquisition. Positive transfer effects of motor learning to different motor systems have been reported frequently (Keele, Jennings, Jones, Caulton, \& Cohen, 1995; Grafton et al., 1998; Kelso \& Zanone, 2002), with most studies addressing and demonstrating positive transfer between both hands (Imamizu \& Shimojo, 1995; Thut et al., 1996; Thut et al., 1997; Sathian \& Zangaladze, 1998; Temprado \& Swinnen, 2005).

However, regarding intermanual transfer, these effects were not always symmetrical but were dependent on the direction of transfer with results being inconsistent whether transfer is better from the dominant to the non-dominant hand (ParkerTaillon \& Kerr, 1989; Parlow \& Dewey, 1991; Thut et al., 1996) or from the non-dominant to the dominant hand (Parlow \& Kinsbourne, 1990; Rosenbaum \& Chaiken, 2001). Additionally, there are also studies that report intermanual symmetry, with transfer to the dominant hand being similar to transfer to the non-dominant hand (Piccirilli, Finali, \& Sciarma, 1989; Mandal, Singh, Asthana, \& Srivastava, 1992; Rand, Hikosaka, Miyachi, Lu, \& Miyashita, 1998; Schulze, Lüders, \& Jäncke, 2002). Intermanual transfer effects have also been found to be dependent on handedness (Stoddard \& Vaid, 1996; Schmidt, Oliveira, Rocha, \& Abreu-Villaca, 2000) and gender (Schmidt et al., 2000), although also for these effects studies have been reported that found no differences between right- and left-handed participants (Mandal et al., 1992; Yamauchi, Imanaka, Nakayama, \& Nishizawa, 2004) or between males and females (Alvis, Ward, \& Dodson, 1989; Parlow \& Kinsbourne, 1990).

The primary goal of the present study was to investigate effects of intermanual transfer to an identical maze and a mirror image of the maze. Significantly greater intermanual transfer to the identical maze compared to the mirror maze would support effector independent coding. An additional goal was to test for possible effects of transfer direction as well as for potential handedness- and gender-related differences.

\section{Methods}

\subsection{Participants}

A total of 24 normal volunteers were tested, 12 right-handed (RH) participants (eight females, four males; mean age 27:11 years) and 12 left-handed (LH) participants (five females, seven males; mean age 31:1 years). All participants were either strongly right- or left-handed, based on the Edinburgh handedness inventory (Raczkowski, Kalat, \& Nebes, 1974). Participants had no prior experience with the experimental task and were naive to the purpose of the study. None of the participants had motor problems. Participants gave their written informed consent and were paid for participation.

\subsection{Material}

Maze designs used in the study are shown in Fig. 1. The mazes were cut out designs and consisted of eight segments, which were connected by intersections. Total (correct) path length of the maze designs was $24 \mathrm{~cm}$. Width of the segments was $0.5 \mathrm{~cm}$ and the depth was $0.15 \mathrm{~cm}$. At the end of each maze segment, participants had two choices, either a correct path, which led to the next choice point, or a dead-end path that had a length of $1 \mathrm{~cm}$. When participants selected a wrong turn at an intersection leading to a dead end, they had to reverse direction to take the correct path to the next choice point. All designs formed closed loops, i.e. the end-point of one loop was the starting-point of the next loop and the designs could be traced continuously.

Participants traced and practiced two versions of the eight-segment maze design, version A and B. Version B was a $90^{\circ}$ rotation of version A. Our earlier research (Van Mier \& Petersen, 2002) has indicated that participants do not notice the rotation but perceived the rotated version as a different maze. One version was used to study the effect of transfer from right to left hand, the other one to study the effect of transfer from left to right hand, with the version of maze presentation counterbalanced across participants. For each maze a mirror image was used to test transfer in the Mirror condition.

Participants traced with a specially designed pen (Maarse, Janssen, \& Dexel, 1988) through the cut-out cardboard designs which were taped to a Calcomp 2500 digitizing tablet. Pen and writing tablet were both connected to an IBM PS2/30 microcomputer allowing accurate recording of the pen movements at a precision of $1 \mathrm{~mm}$ and a frequency of $100 \mathrm{~Hz}$.

\subsection{Procedure}

Participants were seated at a table on a chair that was adjustable in height. The writing tablet was placed on the table and positioned in such a way that a comfortable drawing position was secured. Mazes were taped onto the writing tablet, one at the time. Participants performed the tasks with their eyes closed. Participants had no visual feedback during the tracing sessions and were not allowed to inspect the maze designs visually or tactually. Prior to and during the experiment the mazes would be covered before participants entered the room or before they were allowed to open their eyes during pauses. When mazes needed to be changed between conditions this was done outside the subject's field of vision.

To explain the task and the two-choice principle at intersections, an example maze was shown to the participants. At the start of tracing, the experimenter guided the subject's hand that held the pen, to the starting position in the maze. In all conditions, participants were asked to place the unused hand freely to the side of the writing tablet and keep it in the same position, in order to prevent any interference effects during transfer testing. Research has shown that performance of an unrelated movement by the unused hand during learning (Hicks, Frank, \& Kinsbourne, 1982) or transfer (Parlow \& Dewey, 1991) can prevent intermanual transfer. Participants were instructed to move continuously as quickly and accurately as possible through the maze design with speed and accuracy being equally emphasized. They were told that the first movement was always upwards, that each maze was a loop and that they should try to avoid retracing a path once they experienced a forced stop. Participants were verbally instructed when to start and stop tracing.

Maze tracing performance and the effect of transfer for the right as well as left hand were compared under the following conditions: (1) initial unpracticed maze tracing (Naive), (2) after $10 \mathrm{~min}$ of practice on the same maze (Prac), (3) tracing the mirror maze with the opposite hand (Transfer Mirror), and (4) tracing the identical maze with the opposite hand (Transfer Identical). These four conditions were performed with each hand, so each subject completed a total of eight experimental conditions. The order of hand used, maze version, and of Identical and Mirror transfer conditions was counterbalanced between eight participants. Every other order was repeated in the other four participants.

Before the experimental trials were performed, participants completed two pre-trials in which the identical (Pre-trial Identical) and mirror (Pre-trial Mirror) maze were traced with the same hand used during the transfer trials (see Fig. 1). These trials were included to check for differences related to clockwise and counter clockwise tracing which could account for possible differences between transfer to the identical and mirror maze. Additionally, transfer performance could also be compared to unpracticed performance with the same hand. 
right to left hand transfer

LEFT HAND MAZE TRACING
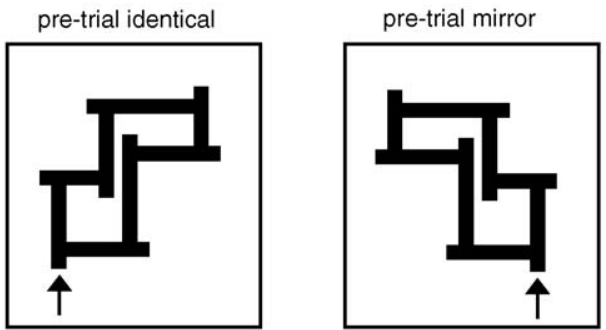

RIGHT HAND MAZE TRACING

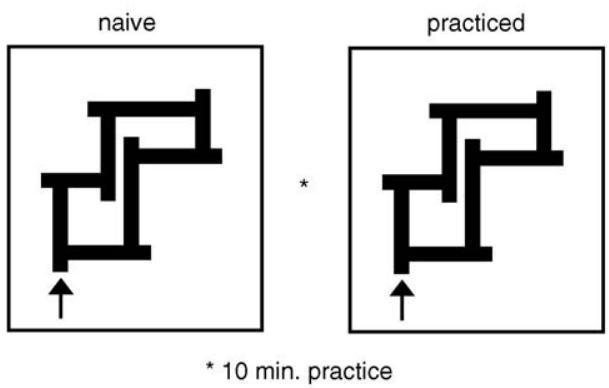

LEFT HAND MAZE TRACING
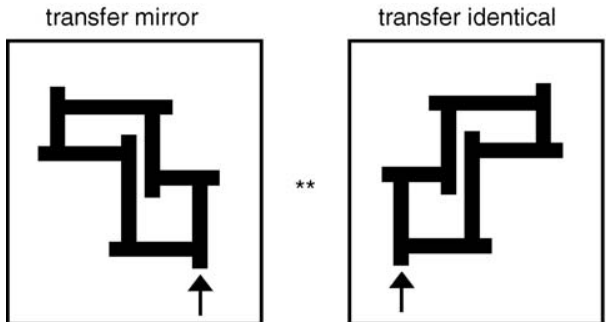

** 2 min. practiced maze tracing with right hand left to right hand transfer

RIGHT HAND MAZE TRACING
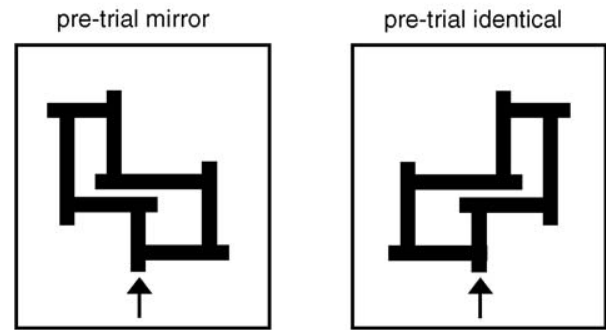

LEFT HAND MAZE TRACING

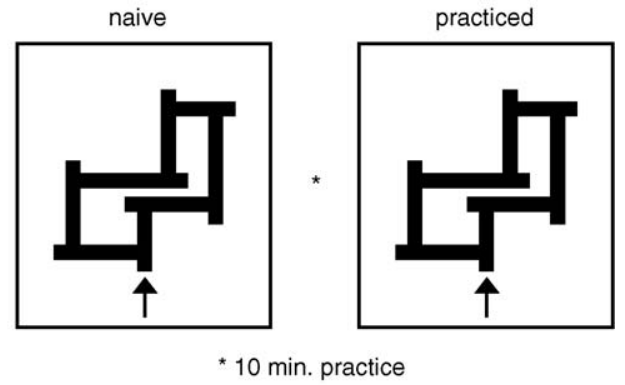

RIGHT HAND MAZE TRACING

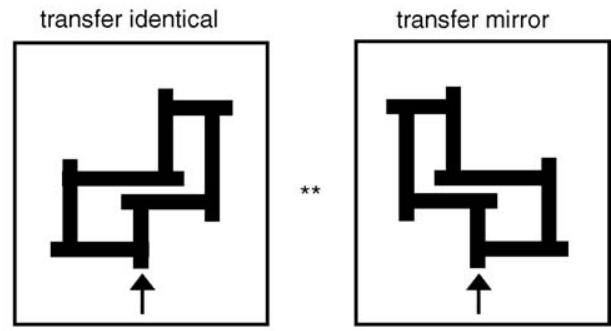

** 2 min. practiced maze tracing with left hand

Fig. 1. Maze designs used in the transfer study. Designs used during transfer from right to left hand are shown on the left, those used during transfer from left to right hand are shown on the right. Arrows indicate the starting position for the tracing of each design. Rh, right hand; Lh, left hand. Note that right hand maze tracing had to be done in a clockwise direction, except during pre-trial tracing of and transfer to the Identical maze, which was done in a counterclockwise direction; left hand maze tracing had to be done in a counterclockwise direction, except during pre-trial tracing of and transfer to the Identical maze, which was done in a clockwise direction. Presentation of maze version and transfer direction was counterbalanced.

During each condition performance was measured for a period of $30 \mathrm{~s}$. Pauses were included every 2 min during the 10 min practice session to prevent participants from slowing down as an effect of fatigue. Transfer was always tested directly after the last practice session was ended. Between the two transfer conditions, participants traced the practiced maze again with the initially trained hand for a period of $2 \mathrm{~min}$ to ensure that performance was at the same level as during the practiced condition. This to guarantee that transfer during the other condition was also measured directly after practiced performance with the opposite hand and that any interference from transfer to the previous condition would be eliminated.

Participants were instructed to trace the mazes in a clockwise direction when the right hand was used, in counterclockwise direction when the left hand was used, with the exception of tracing during pre-trial of and transfer to the Identical maze which had to be done in clockwise direction for left hand tracing and in counterclockwise direction for right hand tracing (see also Fig. 1).

\subsection{Data analysis}

Data records for all conditions were low-pass filtered at $10 \mathrm{~Hz}$ and the drawing trajectories were displayed on the computer screen. The beginning and end of each $30 \mathrm{~s}$ tracing movement was determined by means of an interactive computer program. Mean tracing velocity was calculated for the total period of $30 \mathrm{~s}$. When a dead end path was traced, indicating that an incorrect turn was taken or when a segment was retraced, an error was assigned. The total correct trajectory length per $30 \mathrm{~s}$ was measured as well as the number of loops performed.

The dependent variables of interest in this study were (a) the number of errors per loop, and (b) the mean velocity. For each of these dependent variables a repeated measurement ANOVA was performed with handedness group (rightand left-handed participants) as between factor and transfer direction (transfer from dominant hand to non-dominant hand versus transfer from non-dominant hand to dominant hand) and condition (Naive, Prac, Identical, and Mirror) as within factors (SuperANOVA, version 1.1, Abacus Concepts). When a significant effect of condition was observed, comparisons between the conditions were examined post hoc, for each group and transfer direction. Probabilities were adjusted using the Greenhouse-Geisser epsilon.

To compare transfer performance with unpracticed performance of the same hand, a $2 \times 2 \times 2 \times 2$ (handedness group $\times$ hand $\times$ maze (identical versus mirror) $\times$ condition (pre-trial versus transfer trial)) ANOVA for repeated measurements was performed. Additionally, a separate ANOVA was performed to assess the effect of gender (female vs. male), with gender as between factor 
and transfer direction and condition as within factors. Because the number of males and females was not evenly divided over both handedness groups, data from both right- and left-handed females were compared to those of both rightand left-handed males.

\section{Results}

Since errors were included in the velocity measurement, which might have influenced the data, we additionally measured and analyzed the correct trajectory length. For this variable only the length of the correctly traversed path through the maze was measured. So any distance covered in the dead ends or during retracing was not included. However, since the data analysis of the correct trajectory length showed the same main and post hoc comparison effects as were found for velocity (with only one exception regarding left handed subjects, who did not show a significant difference between naive tracing and transfer to the mirror maze as compared to significant differences found for velocity) trajectory length effects will not further be described and discussed.

\subsection{Errors}

Whenever a participant entered a dead end or retraced a segment (i.e. moved backward), an error was assigned. For each condition the total number of errors made during the $30 \mathrm{~s}$ tracing period was counted, as was the number of loops performed during that period. Because tracing more loops increases the chance of making more errors, the number of errors was divided by the number of loops. There was no significant difference regarding the number of errors between right- and left-handed participants $(F(1,22)=1.25, p=0.28)$. The number of errors per loop for each group and transfer direction is presented in Fig. 2. As can be seen, after having practiced the mazes, participants hardly made any errors, indicating that the participants had learned the mazes adequately. The repeated measures ANOVA with transfer direction and condition as within factors showed that the effect of transfer direction was not significant $(F(1,22)=0.19, p=0.67)$. Transfer from right to left hand did not result in significantly more errors than transfer from left to right hand. As expected, the effect of condition was significant $(F(3,66)=13.64, p<0.001)$. The interaction of transfer direction and condition was not significant, indicating that errors between conditions showed the same conditional pattern regardless if performance and transfer were measured for the left or right hand. None of the other interactions were significant.

Naive and practiced performance was not significantly different between the dominant and non-dominant hand $(p=0.63$ and 0.67 , respectively). The interaction with group was not significant showing that in right- and left-handed participants both hands performed at the same level before and after practice.

To assess differences between conditions, for each group post hoc analyses were performed between the four conditions for each transfer direction. Comparisons between the naive and the practiced condition showed a significant effect of practice for each group and each hand (see Table 1 for $p$ values). As can be seen in this table the difference between the number of errors in
ERRORS PER LOOP
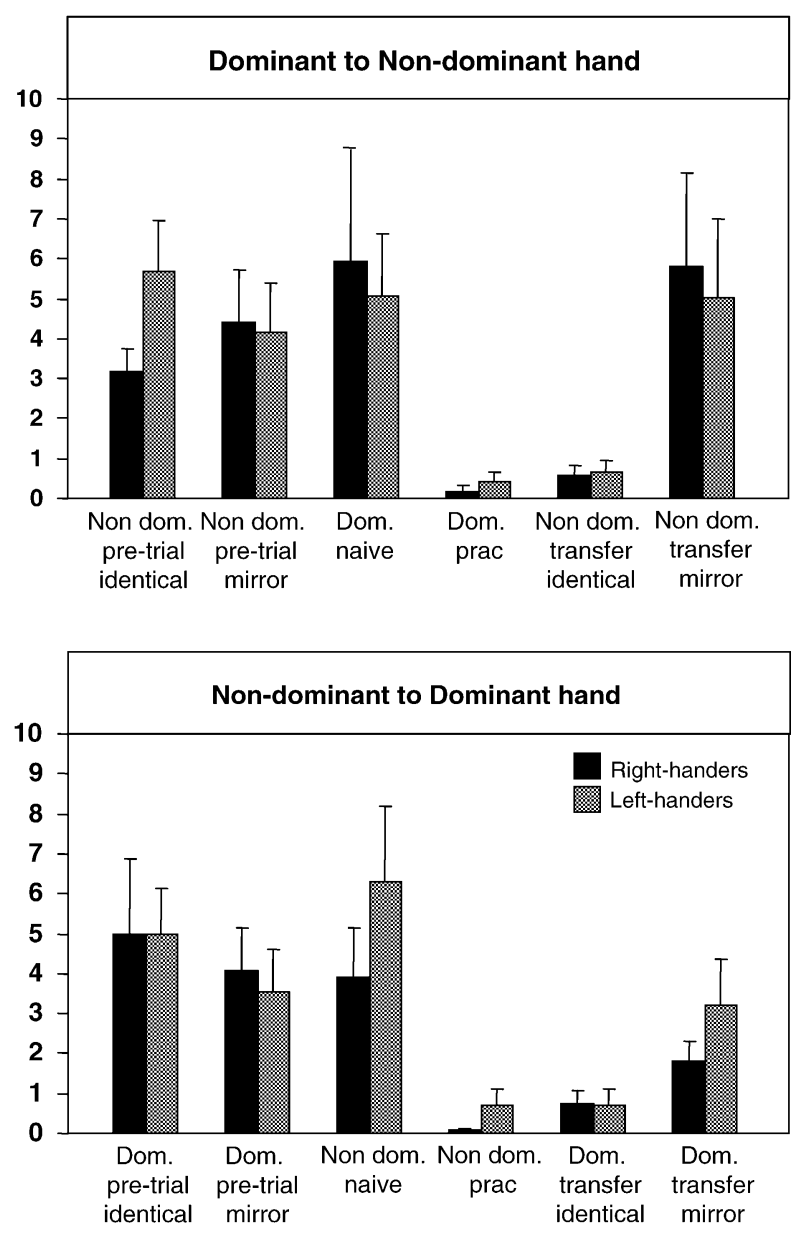

Fig. 2. Number of errors and standard errors for right- and left-handed participants as a function of Transfer direction and Condition. Data from the experimental conditions are presented in black, from pre-trials in grey.

the practiced condition and transfer to the identical maze was not significant. Transfer to the mirror maze resulted only in significantly less accurate performance when right-handed participants transferred to the left hand and when left-handed participants transferred to the right hand, so when participants transferred to their non-dominant hand. This is even more clearly illustrated in Fig. 2. The number of errors per loop during transfer to the mirror maze was not significantly different from the number of errors performed during naive maze tracing while transfer to the identical maze was significantly more accurate than unskilled naive tracing. Levels of significance for the other comparisons are displayed in Table 1.

The analysis comparing same hand performance during the transfer trials and unpracticed pre-trials, showed that the main effect of hand was not significant $(F(1,22)=0.02, p=0.88)$. With the non-dominant hand an average of 3.7 errors was made, with the dominant hand 3.6. A significant main effect of condition was found $(F(1,22)=5.71, p<0.05)$, because more errors were made during the pre-trials (4.4) than during the transfer trials (2.9). The main effect of maze showed a trend $(F(1,22)=4.13$, $p=0.05$ ), with less errors (2.9) made for the identical maze as opposed to the mirror maze (4.3). There was, however, an highly 
Table 1

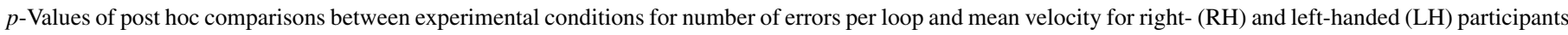
and transfer direction

\begin{tabular}{|c|c|c|c|c|}
\hline \multirow[t]{2}{*}{ Post hoc comparisons } & \multicolumn{2}{|l|}{ Errors } & \multicolumn{2}{|l|}{ Velocity } \\
\hline & $\mathrm{RH}$ & $\mathrm{LH}$ & $\mathrm{RH}$ & $\mathrm{LH}$ \\
\hline \multicolumn{5}{|l|}{ Dominant to non-dominant } \\
\hline Dominant naive vs. dominant prac & $<0.01$ & $<0.01$ & $<0.001$ & $<0.001$ \\
\hline Dominant naive vs. non-dominant transfer identical & $<0.05$ & $<0.05$ & $<0.001$ & $<0.001$ \\
\hline Dominant naive vs. non-dominant transfer mirror & ns & ns & ns & $<0.05$ \\
\hline Dominant prac vs. non-dominant transfer identical & ns & ns & $<0.01$ & ns \\
\hline Dominant prac vs. non-dominant transfer mirror & $<0.05$ & $<0.05$ & $<0.001$ & $<0.01$ \\
\hline Non-dominant transfer identical vs. non-dominant transfer mirror & $<0.05$ & $<0.05$ & $<0.01$ & $<0.05$ \\
\hline \multicolumn{5}{|l|}{ Non-dominant to dominant } \\
\hline Non-dominant naive vs. non-dominant prac & $<0.05$ & $<0.01$ & $<0.001$ & $<0.001$ \\
\hline Non-dominant naive vs. dominant transfer identical & $<0.05$ & $<0.01$ & $<0.001$ & $<0.001$ \\
\hline Non-dominant naive vs. dominant transfer mirror & ns & ns & $<0.01$ & $<0.05$ \\
\hline Non-dominant prac vs. dominant transfer identical & ns & ns & ns & ns \\
\hline Non-dominant prac vs. dominant transfer mirror & ns & ns & $<0.001$ & $<0.01$ \\
\hline Dominant transfer identical vs. dominant transfer mirror & ns & ns & $<0.01$ & $<0.07$ \\
\hline
\end{tabular}

significant interaction between condition and maze $(F(1,22)=$ $14.56, p<0.001)$, due to the fact that differences between identical and mirror maze were only significant for the transfer trials (1.2 versus 4.6 errors for identical and mirror maze) but not for the pre-trials (4.6 versus 4.1 errors, respectively), as can be seen in Fig. 2). Differences between right- and left-handed subjects were not significant $(F(1,22)=0.77, p=0.39)$.

When we analyzed differences between the four pre-trials with hand and maze as within factors and handedness as between factor, performance with both hands, for both mazes and both handedness groups was not significantly different $(p=0.95,0.39$ and 0.72 , respectively) as can be seen in Fig. 2. These results indicate that both hands performed at the same level but also that there were no differences between clockwise and counterclockwise performance.

The ultimate verification of similar performance by both hands was obtained by comparing the errors made during the pre-trials of the identical maze to those made during tracing in the naive conditions, since the same maze was traced in both conditions but by the opposite hands. The analysis showed that tracing with the non-dominant hand was not significantly less accurate than tracing with the dominant hand $(p=0.54)$, nor was tracing during the pre-trials compared to tracing the same maze in the naive conditions $(p=0.57)$. Again right- and left-handers performed similarly $(p=0.45)$.

The additional analysis regarding the effect of gender showed that the number of errors between males and females was not significantly different $(F(1,22)=0.11, p=0.74)$. The effect of gender showed no significant interactions with transfer direction and/or condition.

\subsection{Velocity}

For each condition the mean tracing velocity over each $30 \mathrm{~s}$ period was measured. Fig. 3 illustrates the velocity data for each group and transfer direction. The effect of handedness showed that although right-handers traced the mazes with
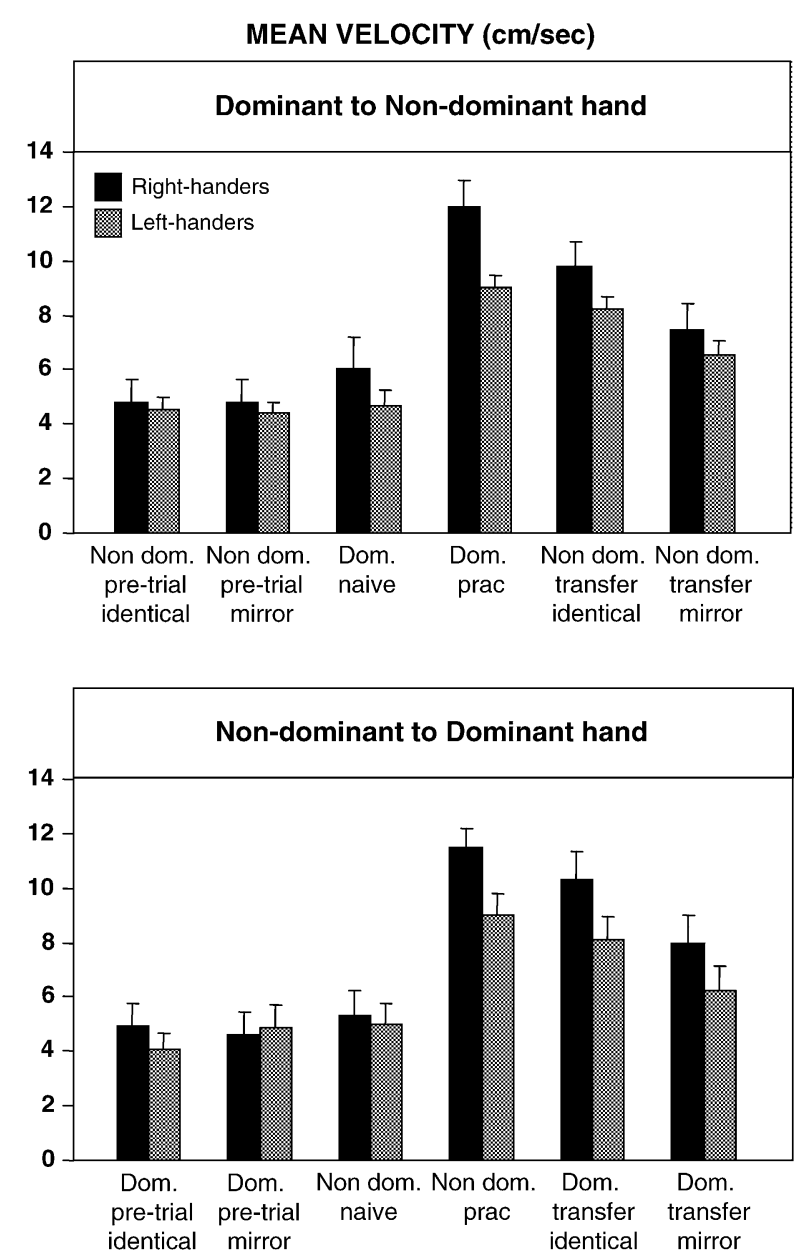

Fig. 3. Mean velocity and standard errors for right- and left-handed participants as a function of Transfer direction and Condition. Data from the experimental conditions are presented in black, from pre-trials in grey. 
higher speed than left-handers, this difference was not significant $(F(1,22)=2.07, p<0.10)$. Also for velocity the main effect of transfer direction was not significant $(F(1,22)=0.03$, $p=0.88)$. Speed of performance was the same regardless if transfer was implemented to the dominant or non-dominant hand. The main effect of condition was highly significant $(F(3,66)=78.97$, $p<0.001)$. The interaction of transfer direction and condition was not significant revealing that conditional effects were the same for performance with and transfer to the dominant and nondominant hand. None of the other interactions was significant, although the interaction of condition and handedness showed a trend $(F(3,66)=2.72, p<0.08)$.

Naive and practiced performance was not significantly different between the dominant and non-dominant hand $(p=0.84$ and 0.67 , respectively). The interaction with group was not significant showing that in right- and left-handed participants both hands performed at the same speed before and after practice.

Differences between the conditions were analyzed by post hoc comparisons. Comparing speed during naive and practiced performance showed that tracing after practice was significantly faster than before for each group and transfer direction (see Table 1). Differences in speed between practiced maze performance and intermanual transfer to the identical maze were not significantly different, except when right-handed participants transferred to the left hand. Comparisons between velocities during practiced tracing and transfer to the mirror maze showed that the speed during the former was significantly faster than during the latter. Naive performance was significantly slower than tracing in the transfer conditions with the exception of transfer to the mirror maze for right-handed participants transferring to the left hand. Participants traced the mazes significantly faster during transfer to the identical maze than to the mirror maze ( $p$ values for all comparisons are given in Table 1).

The analysis comparing same hand performance during the transfer trials and unpracticed pre-trials, showed that the main effect of hand was not significant $(F(1,22)=0.11, p=0.74)$. With the non-dominant hand an average speed of $6.2 \mathrm{~cm} / \mathrm{s}$ was achieved, with the dominant hand a speed of $6.4 \mathrm{~cm} / \mathrm{s}$. A highly significant main effect of condition was found $(F(1,22)=202.14$, $p<0.001)$, because a lower speed was obtained during the pretrials $(4.6 \mathrm{~cm} / \mathrm{s})$ than during the transfer trials $(7.9 \mathrm{~cm} / \mathrm{s})$. The main effect of maze was also very significant $(F(1,22)=16.82$, $p<0.001)$, with a higher speed $(6.8 \mathrm{~cm} / \mathrm{s})$ for the identical maze as opposed to the mirror maze $(5.8 \mathrm{~cm} / \mathrm{s})$. Also for velocity a highly significant interaction between condition and maze was found $(F(1,22)=14.76, p<0.001)$, due to the fact that differences between identical and mirror maze were only significant for the transfer trials $(9.0$ versus $6.9 \mathrm{~cm} / \mathrm{s}$ for identical and mirror maze) but not for the pre-trials (4.6 versus $4.6 \mathrm{~cm} / \mathrm{s}$, respectively), as can be seen in Fig. 3. Again, differences between rightand left-handed subjects were not significant $(F(1,22)=0.36$, $p=0.26$ ).

Analysis of the differences between the four pre-trials with hand and maze as within factors and handedness as between factor, showed that speed was not significantly different between both hands, both mazes and both handedness groups was
( $p=0.99,0.86$ and 0.62 , respectively) as can be seen in Fig. 3 . These results indicate that both hands performed at the same speed but also that there were no differences in velocity between clockwise and counterclockwise tracing.

The ultimate verification of similar performance by both hands was obtained by comparing the speed during the pretrials of the identical maze to the speed during naive tracings, since the same maze was traced in both conditions but by the opposite hands. The analysis showed that tracing speed with the non-dominant hand was almost similar to tracing speed with the dominant hand $(p=0.98)$. However, tracing the same maze in the naive conditions was significantly faster than tracing during the pre-trials $(p<0.01)$. This effect was irrespective of hand, given that the interaction of hand and condition showed no statistical significance $(p=0.83)$. Again right- and left-handers performed at a similar speed $(p=0.37)$.

The additional analysis regarding gender showed that males and females traced the mazes at almost the same speed resulting in a non-significant effect of gender $(F(1,22)=0.02, p=0.89)$. The effects of gender showed no significant interaction with transfer direction and/or condition.

\section{Discussion}

In this study right- and left-handed participants had to find their way through cut-out maze designs by trial and error while they had their eyes closed. Mazes were traced and practiced with the right- as well as the left-hand. After practice, intermanual transfer to an identical maze and a mirror image of the maze was tested. The main findings of this study can be summarized as follows: (1) both right- and left-handed participants showed significantly more intermanual transfer to the identical maze than to the mirror maze; (2) intermanual transfer was symmetrical in both groups, so transfer from dominant to non-dominant hand was not significantly different from transfer from the nondominant to the dominant hand; (3) no significant differences in performance were found between right- and left-handers; (4) no significant effect of gender was observed. Findings 1 and 2 confirm the hypothesis of abstract effector independent performance.

\subsection{Practice and performance prior to transfer}

With respect to practice related findings in the present study, a significant effect of practice was found for both right- and left-handed participants performing with the dominant and nondominant hand. As expected, participants were significantly faster and more accurate after practice. These results replicate earlier findings by our group regarding skill acquisition in the maze task (Van Mier et al., 1993, 1998; Van Mier \& Petersen, 2002; Van Mier et al., 2004; Reithler, van Mier, \& van Breukelen, 2003). The fact that no significant differences were found between the dominant and non-dominant hand before and after practice indicates that this task can be performed equally well by both hands and that the same level of skilled performance was achieved by both hands, making direct comparisons between transfer hands more reliable. 
The finding of almost similar performance for clockwise and counterclockwise tracing of the mazes during the pre-trials shows that differences during transfer could not be related to differences in tracing direction. Additionally, we showed that the non-dominant hand performed at the same level as the dominant hand in the pre-trials, also stressing the fact that the maze task can be performed equally well by both hands.

\subsection{Intermanual transfer}

Regarding our primary goal to examine transfer effects, the results clearly show a significant benefit of transfer to the opposite hand for the identical maze as opposed to the mirror image of the maze. These findings are consistent with the idea that the learned sequence is most likely represented at an abstract level. When participants transfer to the identical maze they can use the exact same movement sequence as practiced before. However, when they transfer to the mirror maze they have to learn a new sequence, resulting in slower and less accurate performance. This was found for transfer from right to left hand as well as from left to right hand, again implying independence from the hand used. As can be seen in Table 1, transfer to the identical maze was almost complete. Differences between practiced performance and transfer to the identical maze were not significant except for right-handed participants, who were faster during dominant hand practice compared to transfer to the nondominant hand. On the other hand differences between practiced tracing and transfer to the mirror maze were significant except for the number of errors when participants transferred to the dominant hand. Results from two other comparisons also suggest that transfer to the identical maze was almost complete while there was less or no transfer to the mirror maze. When comparing naive maze tracing to performance during transfer, it was found that differences regarding transfer to the mirror maze were not significant in most cases, while differences with respect to the identical maze were. A direct comparison between tracing during transfer to the identical maze and to the mirror maze showed that transfer to the former was in most cases faster and more accurate than transfer to the latter. Similar effects of almost complete transfer are also reported by others (Imamizu \& Shimojo, 1995; Sathian \& Zangaladze, 1998).

Our transfer results are consistent with previous findings reported by Stoddard and Vaid (1996), who found significantly more accurate performance in transfer to an identical maze compared to a mirror maze using a finger maze task. Although, they observed a significant interaction of maze and hand used. Participants who transferred to the left hand showed enhanced accuracy on the identical maze, while participants who learned the task with their left hand and transferred to the right hand made significantly less errors on the mirror maze relative to the identical maze. This effect was seen in both right- and left-handed participants. In our study, however, both right- and left-handed participants performed better when they transferred to the identical maze, regardless of the hand used during transfer.

More accurate intermanual transfer when making the same movement instead of a mirror movement is also in agreement with results reported by Rosenbaum and Chaiken (2001). In their study participants had to move a joystick in order to bring a computer-displayed cursor to each of a number of target positions. When the stick moved, the cursor was invisible. After training, participants transferred to the other hand and moved in either the same or opposite (mirror) direction. Moving in the latter direction resulted in significantly greater position errors than to the former. Thut et al. (1997) reported that intermanual transfer of drawing meaningless figures with the right hand after previous practice of mirror-reversals of those figures with the left hand (rTL) was not significantly faster than right hand drawing of a novel figure after previous left-hand practice of an unrelated figure (rOL). These results clearly show an absence of intermanual transfer for the mirror-reversals. It seems that participants not only had to learn a new figure in the rOL condition but also in the rTL condition, where participants had to (re)learn the mirrored figure.

Using PET, regional cerebral blood flow was measured in above-mentioned study when participants performed both tasks. Compared to rOL, rTL showed increased brain activation in bilateral prefrontal areas (BA 9 and 10) with activation in left prefrontal cortex (BA 10) correlating positively with individual rTL performance. On the basis of these findings Thut and colleagues speculate that this prefrontal activation might reflect an inhibitory function. Indeed, participants that showed the smallest left prefrontal activation were faster in drawing the figures in the rTL condition than the rOL condition, while participants with the highest blood flow increases showed the opposite pattern.

Evidence that most likely the abstract movement sequence is stored after practice also comes from a study of Albaret and Thon (1998) in which participants practiced the reproduction of geometrical patterns presented on a video screen, without visual control. They found that intramanual transfer to patterns that were either reduced or enlarged in size resulted in more accurate performance than transfer to rotated mirror images of the patterns. In the former two conditions, by scaling the drawing movements, the same sequence of movements can be performed, while in the latter condition different movement directions have to be performed.

Although above-mentioned findings suggest effector independent and abstract representations in sequential motor tasks, caution is needed generalizing the results to other transfer tasks. The results in our study were observed in participants performing the task at a more or less skilled level. Although participants performed at a level that could be described as advanced learning, their performance was not asymptotic as was the case in a study by Karni et al. (1995). It is possible that once the task is practiced at a level that it can be performed 'automatically', the movement is indeed stored in muscle specific codes, with the primary motor cortex as a likely candidate (Karni et al., 1998; Kleim et al., 2004). At an highly overlearned stage, when the movement is stored in a motor code which, based on the lateralization of the activation in primary motor cortex, would be expected to be specific for the trained hand, no or hardly any transfer to the untrained hand is anticipated. This was indeed observed by Karni et al. (1995). Transfer to the 
other (dominant) hand for the highly trained sequence was not significantly different from transfer for an untrained control sequence.

This view conforms to findings reported by Bapi, Doya and Harner (2000). They tested intramanual transfer effects in a motor sequence-learning paradigm in which participants learned to press ten sets of two keys on a keypad in response to two illuminated squares displayed on a computer monitor. Transfer was tested in a visual as well as in a motor condition. In the visual condition the visual display was kept the same while the keypad was rotated resulting in different finger movements. The opposite was realized in the motor condition in which the visual display was rotated while the keypad was kept the same resulting in similar finger movements. Transfer performance was tested during early, intermediate and late stages of practice. There were no significant differences between the transfer conditions during early learning, suggesting an effector independent sequence representation. However, in the late practice stage, performance was significantly better during transfer in the motor condition confirming the existence of an effector dependent sequence representation.

Consistent with this pattern of result are two studies reported by Rand et al. (1998, 2000). Studying intermanual transfer on a sequential button-pressing task in monkeys, Rand et al. (1998) observed that performance using the opposite hand was significantly slower and less accurate than practiced performance with the original hand, but it was, however, significantly faster and more accurate than new performance with the original hand. Intermanual transfer in the monkeys was tested after they had practiced the sequence for several weeks. As the authors state, it is very likely that in this study transfer was tested at the transition phase when the movement representation changes from mainly effector independent to becoming more specific to the motor system used during acquisition and practice. In a follow up study using the same task, Rand et al. (2000) studied intermanual transfer in monkeys during early learning. They found complete transfer to the unpracticed hand for the number of errors. With respect to performance time, transfer was observed, however, this was not complete.

Contrary to the above-mentioned results are findings reported by Japikse, Negash, Howard and Howard (2003). In her study, participants showed almost complete transfer from the dominant right hand to the non-dominant left hand in a probabilistic alternating serial reaction time after extensive practice (at least 1000 pattern repetitions). However, the fact that within an eight trial sequence, only the $1 \mathrm{st}, 3 \mathrm{rd}$, 5 th and 7 th trial were repeated while the other trials were random might have prevented pure effector specific learning.

Differences in the level of practice or training might indeed explain the contradictory results reported in the literature on transfer of learning. While differences are reported for transfer to an identical or mirror maze depending on the hand used during acquisition (Stoddard and Vaid, 1996) transfer in this study was measured after successful completion of one error-free run on non-visual tactile learning of a finger maze. Transfer was measured during very early learning, after, on average, eight runs through the maze.
As is apparent from above-mentioned studies, results obtained in transfer tasks seem to be dependent on the amount of and level of skill obtained during pre-transfer practice. While highly over learned movements are most likely represented at an effector-specific level, effector independent transfer can be expected when learning is not complete.

\subsection{Effects of transfer direction, handedness and gender}

The second goal of our study was to examine the influence of the direction of transfer, handedness and gender in the sequential maze task. None of these factors had a significant effect on speed and accuracy.

Studies involving right-handed participants often have reported asymmetric intermanual transfer in movement tasks, with either greater transfer from the left non-dominant hand to the right dominant hand (Ammons \& Ammons, 1951; Parlow \& Kinsbourne, 1990; Lavrysen et al., 2003) or from the right dominant to the left non-dominant hand (Parlow \& Dewey, 1991; Halsband, 1992; Thut et al., 1996). However there are also studies that reported no transfer (Teixeira, 1993), or equal transfer to both directions (Imamizu \& Shimojo, 1995; Palmer \& Meyer, 2000), as we did in our study. Others have shown similar overall transfer for right and left hand in visuo-motor rotations, although different features of movement transferred in different directions (Sainburg \& Wang, 2002).

(A)symmetry of intermanual transfer seems to be dependent on the task used as illustrated by the diversity of results reported in above-mentioned studies employing different tasks and paradigms. In the studies mentioned above showing a benefit for intermanual transfer from the dominant to the non-dominant hand (Parlow \& Dewey, 1991; Halsband, 1992; Thut et al., 1996) performance before transfer was always more proficient for the dominant hand than the non-dominant hand. Thut et al. (1996) reported that training with the left hand negatively influenced performance by the right hand, meaning that transfer to the right dominant hand was inferior to performance with this hand before learning with the left hand. In their follow up study using PET, they showed that this negative transfer effect correlated with brain activation in the left prefrontal cortex (BA 10) (Thut et al., 1997). Thut et al. $(1996,1997)$ used the same sort of stimuli as previously used by Halsband in her 1992 paper. Halsband looked at isochrony during training of new ideograms at different sizes with both hands and transfer to the untrained hands. Isochrony is the principle that in over learned motor tasks movement time stays invariant irrespective of movement amplitude (Viviani \& Terzuolo, 1980). Halsband found that after training isochrony was achieved by both hands. However, significant transfer was only found from right hand to left hand. This asymmetric effect in transfer in the above mentioned studies by Thut et al. (1996) and Halsband (1992) might be due to the fact that performance by both hands was different with significantly faster and more accurate performance of the right hand compared to the left hand in the study by Thut et al. (1996) and faster performance and acquisition of isochrony with the right hand in the study by Halsband (1992). Superior performance of the right hand before transfer to the left hand suggests that only after right hand learning the 
abstract features of the ideogram were stored in such a way that the left hand could access them.

As was the case in our previous study (Van Mier et al., 1998), in the present study the maze task was performed at a similar level with both hands. None of the main effects involving comparisons between both hands was statistically significant. This might explain the effect of comparable transfer in both directions. Analogous performance by both hands before transfer appears to predict that transfer to the identical maze would be complete, resulting in performance that would not be significantly different from performance before transfer. This was indeed observed in our study. However, please note that righthanded subjects showed greater transfer from non-dominant to dominant hand for the identical maze regarding velocity. With respect to accuracy, right-handed subjects also made more errors when transferring to left hand tracing of the mirror maze compared to left hand tracing of the naive maze. Although these effects were not significant, the data give a hint of some asymmetrical transfer in this group, comparable to the effects reported by Thut et al. (1996) and Halsband (1992).

While most experiments concerning intermanual transfer included right-handed participants (Halsband, 1992; Thut et al., 1996, 1997; Sathian \& Zangaladze, 1998), only a few studies compared performance in both right- and left-handed participants (Stoddard \& Vaid, 1996; Velay \& Benoit-Dubrocard, 1999; Palmer \& Meyer, 2000; Schmidt et al., 2000). When groups were compared based on their handedness, most studies report no differences between right- and left-handed groups (Velay \& Benoit-Dubrocard, 1999) as was the case in our study.

The reported asymmetry of transfer in studies including right- and left-handed participants (Parlow \& Kinsbourne, 1990; Lavrysen et al., 2003) was not replicated in our study. Our findings are in agreement with results published by others (Piccirilli et al., 1989; Mandal et al., 1992; Stoddard \& Vaid, 1996).

With respect to gender effects, men and women performed our maze task at the same level, corroborating findings by Alvis et al. (1989) and Parlow and Kinsbourne (1990). Because the number of left and right-handed women and men was not equally divided in each group, we were not able to test the effect of handedness as well.

\section{Conclusion}

Taking together our results point toward an abstract effector independent representation in the sequential maze task. The finding that transfer was more or less complete to the identical maze compared to significantly less transfer to a mirror image of the maze suggests that an abstract overall pattern of the maze is stored. The fact that transfer was similar from dominant to nondominant hand and vice versa in both right- and left-handed participants stresses complete effector independence in our task with respect to the amount of practice given.

Differences between the effects of transfer found across studies seem to be related to the amount and level of practice achieved, with almost complete transfer during early learning phases when effector independent representations are stored to less and less transfer after extensive practice, when the representation becomes highly effector dependent.

\section{References}

Albaret, J. M., \& Thon, B. (1998). Differential effects of task complexity on contextual interference in a drawing task. Acta Psychologica, 100, 9-24.

Alvis, G. R., Ward, J. P., \& Dodson, D. L. (1989). Equivalence of male and female performance on a tactuospatial maze. Bulletin of the Psychonomic Society, 27, 29-30.

Ammons, R. B., \& Ammons, C. H. (1951). Bilateral transfer of rotary-pursuit skill. American Psychologist, 6, 294.

Bapi, R. S., Doya, K., \& Harner, A. M. (2000). Evidence for effector independent and dependent representations and their differential time course of acquisition during motor sequence learning. Experimental Brain Research, 132, 149-162.

Cisek, P., Crammond, D. J., \& Kalaska, J. F. (2003). Neural activity in primary motor and dorsal premotor cortex in reaching tasks with the contralateral versus ipsilateral arm. Journal of Neurophysiology, 89, 922942.

Doyon, J., Penhune, V., \& Ungerleider, L. G. (2003). Distinct contribution of the cortico-striatal and cortico-cerebellar systems to motor skill learning. Neuropsychologia, 41, 252-262.

Fischman, M. G., \& Lim, C.-H. (1991). Influence of extended practice on programming time, movement time, and transfer in simple target-striking responses. Journal of Motor Behavior, 23, 39-50.

Grafton, S. T., Hazeltine, E., \& Ivry, R. B. (1998). Abstract and effectorspecific representations of motor sequences identified with PET. Journal of Neuroscience, 18, 9420-9428.

Grafton, S. T., Hazeltine, E., \& Ivry, R. B. (2002). Motor sequence learning with the nondominant left hand. A PET functional imaging study. Experimental Brain Research, 146, 369-378.

Halsband, U. (1992). Left hemisphere preponderance in trajectorial learning. Neuroreport, 3, 397-400.

Hazeltine, E. (2001). Ipsilateral sensorimotor regions and motor sequence learning. Trends in Cognitive Science, 5, 281-282.

Hicks, R. E., Frank, J. M., \& Kinsbourne, M. (1982). The locus of bimanual skill transfer. Journal of General Psychology, 107, 277-281.

Imamizu, H., \& Shimojo, S. (1995). The locus of visual-motor learning at the task or manipulator level: Implications from intermanual transfer. Journal of Experimental Psychology: Human Perception and Performance, 21, 719-733.

Japikse, K. C., Negash, S., Howard, J. H., Jr., \& Howard, D. V. (2003). Intermanual transfer of procedural learning after extended practice of probabilistic sequences. Experimental Brain Research, 148, 38-49.

Karni, A., Meyer, G., Jezzard, P., Adams, M. M., Turner, R., \& Ungerleider, L. G. (1995). Functional MRI evidence for adult motor cortex plasticity during motor skill learning. Nature, 377, 155-158.

Karni, A., Meyer, G., Rey-Hipolito, C., Jezzard, P., Adams, M. M., Turner, R., \& Ungerleider, L. G. (1998). The acquisition of skilled motor performance: Fast and slow experience-driven changes in primary motor cortex. Proceedings of the National Academy of Science USA, 95, 861-868.

Keele, S. W., Jennings, P., Jones, S., Caulton, D., \& Cohen, A. (1995). On the modularity of sequence representation. Journal of Motor Behavior, $27,17-30$.

Kelso, J. A., \& Zanone, P. G. (2002). Coordination dynamics of learning and transfer across different effector systems. Journal of Experimental Psychology: Human Perception and Performance, 28, 776-797.

Kleim, J. A., Hogg, T. M., VandenBerg, P. M., Cooper, N. R., Bruneau, R., \& Remple, M. (2004). Cortical synaptogenesis and motor map reorganization occur during late, but not early, phase of motor skill learning. Journal of Neuroscience, 24, 628-633.

Lavrysen, A., Helsen, W. F., Tremblay, L., Elliott, D., Adam, J. J., Feys, P., \& Buekers, M. J. (2003). The control of sequential aiming movements: The influence of practice and manual asymmetries on the one-target advantage. Cortex, 39, 307-325.

Maarse, F. J., Janssen, H. J. J., \& Dexel, F. (1988). A special pen for an XY-tablet. In F. J. Maarse, L. J. M. Mulder, W. P. B. Sjouw, \& A. E. 
Akkerman (Eds.), Computers in Psychology; Methods, Instrumentation, and Psychodiagnostics (pp. 133-139). Amsterdam: Swets and Zeitlinger.

Mandal, M. K., Singh, S. K., Asthana, H. S., \& Srivastava, P. (1992). Bilateral transfer deficit in schizophrenia. Comprehensive Psychiatry, 33, 319-324.

Palmer, C., \& Meyer, R. K. (2000). Conceptual and motor learning in music performance. Psychological Science, 11, 63-68.

Parker-Taillon, D., \& Kerr, R. (1989). Manual asymmetries within the performance of a complex motor task. Human Movement Science, 8, 33-44.

Parlow, S. E., \& Kinsbourne, M. (1990). Asymmetrical transfer of braille acquisition between hands. Brain and Language, 39, 319-330.

Parlow, S. E., \& Dewey, D. (1991). The temporal locus of transfer of training between hands: An interference study. Behavioral Brain Research, 46, $1-8$.

Petersen, S. E., van Mier, H., Fiez, J. A., \& Raichle, M. E. (1998). The effects of practice on the functional anatomy of task performance. Proceedings of the National Academy of Science USA, 95, 853-860.

Piccirilli, M., Finali, G., \& Sciarma, T. (1989). Negative evidence of difference between right- and left-handers in interhemispheric transfer of information. Neuropsychologia, 27, 1023-1026.

Raczkowski, D., Kalat, J. W., \& Nebes, R. (1974). Reliability and validity of some handedness questionnaire items. Neuropsychologia, 6, 43-47.

Raibert, M.H., 1977. Motor Control and Learning by the State Space model. Technical Report AI-TR-439, MIT, Artificial Intelligence Laboratory, Cambridge, MA.

Rand, M. K., Hikosaka, O., Miyachi, S., Lu, X., \& Miyashita, K. (1998). Characteristics of a long-term procedural skill in the monkey. Experimental Brain Research, 118, 293-297.

Rand, M. K., Hikosaka, O., Miyachi, S., Lu, X., Nakamura, K., Kitaguchi, K., \& Shimo, Y. (2000). Characteristics of sequential movements during early learning period in monkeys. Experimental Brain Research, 131, 293-304.

Rao, S. M., Binder, J. R., Bandettiini, P. A., Hammeke, T. A., Yetkin, F. Z., Jesmanowicz, A., Lisk, L. M., Morris, G. L., Mueller, W. M., Estkowski, L. D., Wong, E. C., Haughton, V. M., \& Hyde, J. S. (1993). Functional magnetic resonance imaging of complex human movements. Neurology, 43, 2311-2318.

Reithler, J., van Mier, H., \& van Breukelen, G. (2003). Effects of mental and physical practice in a sequential motor learning task. In H. L. Teulings \& A. W. A. van Gemmert (Eds.), Proceedings of the 11th Conference of the International Graphonomics Society (pp. 115-118). Tempe: International Graphonomics Society.

Rosenbaum, D. A., Kenny, S. B., \& Derr, M. A. (1983). Hierarchical control of rapid movement sequences. Journal of Experimental Psychology: Human Perception and Performance, 9, 86-102.

Rosenbaum, D. A., \& Chaiken, S. R. (2001). Frames of reference in perceptual-motor learning: Evidence from a blind manual positioning task. Psychological Research, 65, 119-127.

Sainburg, R. L., \& Wang, J. (2002). Interlimb transfer of visuomotor rotations: Independence of direction and final position information. Experimental Brain Research, 145, 437-447.

Sakai, K., Hikosaka, O., Miyauchi, S., Takino, R., Sasaki, Y., \& Pütz, B. (1998). Transition of brain activations from frontal to parietal areas in visuomotor sequence learning. Journal of Neuroscience, 18, 1827-1840.

Sathian, K., \& Zangaladze, A. (1998). Perceptual learning in tactile hyperacuity: Complete intermanual transfer but limited retention. Experimental Brain Research, 118, 131-134.

Schmidt, R. A., \& Lee, T. D. (1999). Motor control and learning: A behavioral emphasis (3rd ed.). Champaign, IL: Human Kinetics.

Schmidt, S. L., Oliveira, R. M., Rocha, F. R., \& Abreu-Villaca, Y. (2000). Influences of handedness and gender on the grooved pegboard test. Brain and Cognition, 44, 445-454.
Schulze, K., Lüders, E., \& Jäncke, L. (2002). Intermanual transfer in a simple motor task. Cortex, 38, 805-815.

Stoddard, J., \& Vaid, J. (1996). Asymmetries in intermanual transfer of maze learning in right- and left-handed adults. Neuropsychologia, 34, 605608 .

Tanji, J., Okano, K., \& Sato, K. C. (1988). Neuronal activity in cortical motor areas related to ipsilateral, contralateral, and bilateral digit movements of the monkey. Journal of Neurophysiology, 60, 325-343.

Tanji, J., \& Shima, K. (1994). Role for supplementary motor area cells in planning several movements ahead. Nature, 371, 413-416.

Temprado, J. J., \& Swinnen, S. P. (2005). Dynamics of learning and transfer of muscular and spatial relative phase in bimanual coordination: Evidence for abstract directional codes. Experimental Brain Research, 160, $180-188$

Teixeira, L. A. (1993). Bilateral transfer of learning: The effector side in focus. Journal of Human Movement Studies, 25, 243-253.

Thut, G., Cook, N. D., Regard, M., Leenders, K. L., Halsband, U., \& Landis, T. (1996). Intermanual transfer of proximal and distal motor engrams in humans. Experimental Brain Research, 108, 321-327.

Thut, G., Halsband, U., Roelcke, U., Nienhusmeier, M., Missimer, J., Maquire, R. P., Regard, M., Landis, Th., \& Leenders, K. L. (1997). Intermanual transfer of training: Blood flow correlates in the human brain. Behavioral Brain Research, 89, 129-134.

Tresilian, J. R. (1999). Abstract levels of motor control in prehension: Normal and pathological performance. Human Movement Science, 18, 219-239.

Van Mier, H., \& Hulstijn, W. (1993). The effects of motor complexity and practice on initiation time in writing and drawing. Acta Psychologica, 84, 231-251.

Van Mier, H., Hulstijn, W., \& Petersen, S. E. (1993). Changes in motor planning during the acquisition of movement patterns in a continuous task. Acta Psychologica, 82, 291-312.

Van Mier, H., Tempel, L. W., Perlmutter, J. S., Raichle, M. E., \& Petersen, S. E. (1998). Changes in brain activity during motor learning measured with PET: Effects of hand of performance and practice. Journal of Neurophysiology, 80, 2177-2200.

Van Mier, H. (2000). Human learning. In A. W. Toga \& J. C. Mazziotta (Eds.), Brain Mapping: The Systems (pp. 605-621). San Diego: Academic Press.

Van Mier, H., \& Petersen, S. E. (2002). The role of the cerebellum in motor cognition. Annals of the New York Academy of Sciences, 978, 334 353

Van Mier, H., Perlmutter, J. S., \& Petersen, S. E. (2004). Functional changes in brain activity during acquisition and practice of movement sequences. Motor Control, 8, 500-520.

Velay, J. L., \& Benoit-Dubrocard, S. (1999). Hemispheric asymmetry and interhemispheric transfer in reaching programming. Neuropsychologia, 37, 895-903.

Verwey, W. B., Lammens, R., \& Honk, J. (2002). On the role of the SMA in the discrete sequence production task: A TMS study. Neuropsychologia, 40, 1268-1276.

Viviani, P., \& Terzuolo, C. (1980). Space-time invariance in learned motor skills. In G. E. Stelmach \& J. Requin (Eds.), Tutorials in Motor Behavior (pp. 525-533). Amsterdam: North Holland.

Wright, C. E. (1990). Generalized motor programs: Reexamining claims of effector independence in writing. In M. Jeannerod (Ed.), Attention and Performance XIII; Motor Representation and Control (pp. 294-320). Hillsdale, NJ: Lawrence Erlbaum Associates.

Yamauchi, M., Imanaka, K., Nakayama, M., \& Nishizawa, S. (2004). Lateral difference and interhemispheric transfer on arm-positioning movement between right- and left-handers. Perceptual and Motor Skills, 98, 1199-1209. 